\title{
TECHNIQUES FOR DETECTION OF SOLITARY Pulmonary Nodules in Human Lung AND THEIR ClassificATIONS -A SURVEY
}

\author{
Bhavanishankar . $\mathrm{K}^{1}$ and Dr. M.V.Sudhamani ${ }^{2}$ \\ ${ }^{1}$ Asst. Prof. Department of CSE \\ ${ }^{2}$ Prof. and Head, Department of ISE \\ RNS Institute of Technology, Bengaluru, India
}

\begin{abstract}
Lung cancer is proving to be a catastrophic threat to the mankind and is main cause of deaths among other cancer related casualties. The presence of solitary pulmonary nodules in human lungs in the form of benign or malignant determines the gravity of lung ailment. This survey focusses on different techniques used to detect and classify the lung nodules which in turn will assist the domain experts for better diagnosis. Among many imaging modalities Computed Tomography (CT) being the most sought after because of its high resolution, isotropic acquisition which helps in locating the lung lesions. Since the volume of the CT scans are very large, Computer Aided Detection/Diagnosis (CAD/x) has more advantages in addition to manual interpretation with respect to speed and accuracy. This paper attempts to summarize various methods that have been proposed by several authors over the years of their research.
\end{abstract}

\section{KEYWORDS}

Lung, Solitary pulmonary nodule, Sgmentation, Tresholding.

\section{INTRODUCTION}

Human lungs are pair of large, spongy organs that help to respire which constitutes the respiratory system. The main chore of this system is to make fresh air (oxygen) into and get waste gases (carbon dioxide) out of the body [1]. The windpipe - trachea, transmits inhaled air in to the lungs through tubular branches-bronchi, which divides further in to smaller branches called bronchioles. The bronchioles ultimately culminates in bunch of air sacs called alveoli, where oxygen from the air is absorbed in to the blood. Carbon dioxide the waste product of metabolism moves from blood to alveoli where it is exhaled

Cancer is when cells in the body begin to grow out of control. In cancerous cells the DNA which is present in every cell is not repaired after damage rather these cells grow and form new abnormal cells. Among many other cancers, Lung cancer is an aggressive and heterogeneous disease and is the leading cause of cancer related deaths resulting in more deaths than other cancers combined. As per the statistics of American Cancer Society, lung cancer is the major cause of the cancer related death in USA. The estimates of new cases and deaths in 2012 are 2, 26,160 and 1, 60,340 respectively. In 2013 the estimated new cases and deaths are 2, 28,190 and $1,59,480$ [1] respectively. In India approximately 63,000 lung cancer cases are reported every year. When a person has lung cancer, then they have abnormal cells that cluster together to form a tumour (nodule). Not all tumours are cancerous. These non- cancerous tumours are called benign 
nodules. The other cancerous nodules that grow without order, control and obliterate the healthy lung tissues around them are called malignant nodules.

Lung cancer is classified into two types (i) Small Cell Lung Cancer (SCLC) which constitutes $10 \%$ to $15 \%$ of all lung cancers and (ii) Non-Small Cell Lung Cancer (NSCLC) which shares $80 \%$ to $90 \%$ of lung cancers. Though early stages of NSCLC - stages I, II and IIIA, can be treated with remedial lung resection, advanced stages- Stages IIIB and IV, have very gloomy prognosis [2].

A Computer Aided Detection System (CAD) is one of the principal research streams in medical imaging and diagnostic radiology. A well-developed CAD helps in processing image for detection and extraction of abnormalities and also aids in classification of image features between normal and abnormal. In most of the cases CAD offers a very useful second opinion when radiologist examine patient at cancer $\mathrm{CT}$ screenings. A CAD system is instrumental in reducing the number of false negative diagnosis [3].The success of a CAD system is measured in terms of accuracy in diagnosis, speed and its degree of automation.

Amid various non-invasive medical imaging modalities such as X-RAY, Magnetic Resonance Imaging (MRI), Positron Emission tomography (PET), PET-CT, the Computed Tomography (CT) is widely used for spotting and diagnosing lung nodules. Since the invention in 1972 by G.N.Hounsfiled, the advancement in CT imaging has contributed in a great deal to the field of Pathology. CT has been influential in increasing the survival rate by diagnosing the life threatening ailments, mainly the cancer [4]. Recent CT scanners exhibit isotropic acquisition of whole chest with very high resolution within a single breath hold.

A typical lung nodule detection scheme is shown in Figure.1, which comprises of the following sequences of processing steps. Image Acquisition, Image Enhancement, Lung Parenchyma Segmentation, Candidate nodule Detection, False Positive Reduction and Nodule Classification. These steps are explained in the following sections.

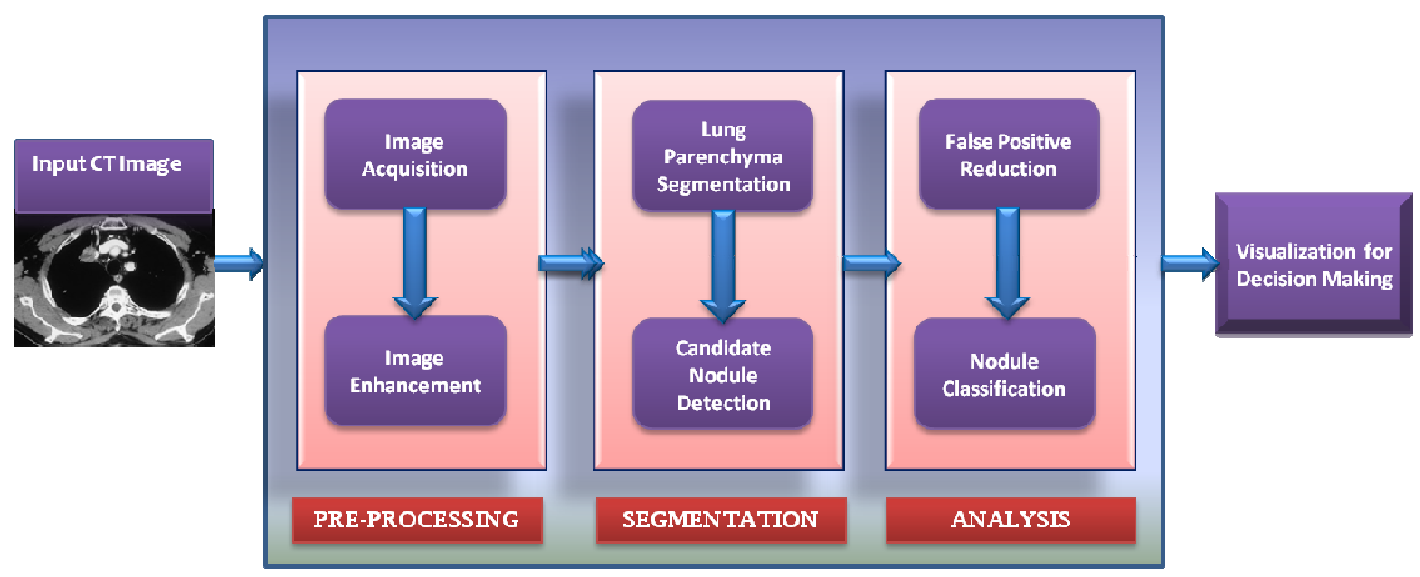

Figure 1. Processing steps in typical Lung nodule detection scheme

\section{IMAGE ACQUISITION}

To design a CAD for lung nodule detection system, CT images are well preferred as offers visualization of low contrast or small volume nodules by diminishing the slice thickness. Lung CT images can be acquired from publicly available databases namely Early Lung Cancer Action 
International Journal on Cybernetics \& Informatics (IJCI) Vol. 4, No. 1, February 2015

Program (ELCAP) [5], Lung Image Database Consortium (LIDC) [6] or Medical Image Database [7]. As per the literature there are other imaging techniques and many private databases used by the researchers, which are obtained from private hospitals.

\section{Preprocessing}

Since the Digital Imaging and Communications in Medicine (DICOM) images with noise uses the reconstruction method to enhance resolution denoising these original images is necessary. Preprocessing of an image refers to the procedure of enhancing the quality and interpretability of the input lung image by reducing the noise and unwanted artefacts. In lung CT scans, preprocessing improves the visibility of pulmonary nodules. Types of filters used in preprocessing phase are Laplacian of Gaussian filter (LOG), Ring Average filters, Median filters, Morphological filters, Selective Enhancement Filter and so on.

\subsection{Selective Enhancement filters}

Various types of selective enhancement filters are used to enhance blob like structures and to suppress vessel like structures by [8] [9] [10] [11] and [12] recommended a selective enhancement filter to enhance dot like objects and to repress lung vessels. Cylindrical and spherical filters were combined for a better visualization of nodules by [13].

\subsection{LoG filter}

The Laplacian of Guassian (LoG) filter is preferred in enhancing blob like structures whose intensity is differs from that of background. [14] used LoG filters to enhance the input image. [15] and [16] recommended LoG filter for enhancement.

\subsection{Median filter}

Median filter was used by [17] and [18] to remove the noise from the image. The advantage of this filter is that it doesn't distort the edges.

\subsection{Other filters}

Prewitt filter was used by [19] to enhance the horizontal edges. 3D filter was 1. introduced by [20] to orient the Ground Glass Opacity nodules. [21] Compared several filters and used a linear smoothing filter called adaptive Weiner filter for studies. To denoise and preserve the spherical structures 3-D Coherence-Enhancing Diffusion (CED) filter is used on the acquired CT images by [22]. Bilateral filters are used to remove the noise in the image by [23]. 2-D Mutiscale filters are used by [24] to search for blob like geometrical structures in image. A summary of the enhancement methods and a note of remarks are given in the Table 1. 
International Journal on Cybernetics \& Informatics (IJCI) Vol. 4, No. 1, February 2015

Table 1. Summary of various enhancement methods

\begin{tabular}{|l|l|l|}
\hline Study & Method & Features/Results \\
\hline Takahiro et al. [8] & Selective Enhancement Filters & $\begin{array}{l}\text { Enhance blob like structures, } \\
\text { Suppress vessel like } \\
\text { structures }\end{array}$ \\
\hline Sun et al.[11] & Selective Enhancement Filters & $\begin{array}{l}\text { Enhance dot like objects, } \\
\text { Repress lung vessels }\end{array}$ \\
\hline Chen et al. [13] & $\begin{array}{l}\text { Combination of Cylindrical } \\
\text { and Spherical Filters }\end{array}$ & $\begin{array}{l}\text { Better visualization of } \\
\text { nodules }\end{array}$ \\
\hline Vijaya Kishore et al. [19] & Prewitt Filter & Enhance horizontal edges \\
\hline Oda et al. [20] & 3D Filter & $\begin{array}{l}\text { Orientation of the Ground } \\
\text { Glass Opacity nodules }\end{array}$ \\
\hline Sergei V. Fotin et al. [14] & LoG filter & $\begin{array}{l}\text { Enhance the blob like } \\
\text { structure }\end{array}$ \\
\hline Hong Shao et al. [21] & Adaptive Weiner Filter & Denoising, smoothing \\
\hline Shingo et al.[24] & 2D Mutiscale Filter & $\begin{array}{l}\text { Search and enhance blob like } \\
\text { structure }\end{array}$ \\
\hline
\end{tabular}

\section{Lung Parenchyma Segmentation}

Image segmentation is a technique of partitioning an image in to multiple regions of the lung refers to the process of extracting the lung region from other anatomical parts of the body in chest CT images. This process plays a vital role in nodule detection by improving accuracy and precision that helps in early diagnosis of lung cancer. An accurate segmentation will reduce the computational cost of detection.

In CT image of a lung, the anatomical structures that may require segmentation are lungs themselves, the airways, the vessels, lung lobes. Segmentation is a complex activity due to pulmonary structures of similar densities namely arteries, veins, bronchioles and different scanners used. Numerous publications have addressed the issue of segmentation of lungs. The proposed techniques are measures with respect to accuracy, processing time and level of automation. Mostly used segmentation techniques can be classified as methods based on thresholding, deformable boundaries, edge detection and shape models.

Lung is a sack of air in the body which exhibit as darker regions in CT images compared to other parts of the chest. This fact has motivated the researchers to explore an optimum threshold which separates the lung from other tissues. The majority of the research in the segmentation revolved around techniques such as thresholding, multiple thresholding, optimal thresholding, adaptive thresholding, region growing, graph cuts, active contour model, hybrid segmentation, fuzzy c means clustering, morphological operations and so on.

\subsection{Thresholding}

In thresholding techniques selecting the threshold level and in region growing approach selecting a seed point has a greater impact on segmentation outcome. The recent methods use model based and optimization methods as compared to earlier heuristic based approaches. [25] obtained a threshold that separates the lung from other tissues. Region growing followed by Connected Component Analysis method was proposed by [26] to extract the lung region. [27] proposed an accurate segmentation method with four step. (1) Extracting the airway from lung region (2)Removal of pulmonary arterial and venous vessel trees by finding a suitable threshold (3) 
International Journal on Cybernetics \& Informatics (IJCI) Vol. 4, No. 1, February 2015

Using a largest threshold to separate right and left lung (4) Morphological smoothing of lung boundary . [28] performed the segmentation using histogram analysis followed by Connected Component Labeling (CCL) and morphological closing operations to smother the segmented lung.

The accuracy of threshold based segmentation depends upon image acquisition protocol and acquisition type. In most of the cases the concentration of pulmonary constructs, say arteries, veins, bronchi are very much near to the concentration of chest tissues. To overcome this inhomogeneity in the densities in the territory of lung, further rigorous post processing is required.

\subsection{Deformable boundary models}

Deformable boundary models such as active contours, level sets were also used in segmentation. Active contours or snakes are capable of extracting the Region of Interest. A parametric deformable model was used [29] to separate lung region. The deformable models had serious problems pertaining to speed, initialization and convergence with boundaries.

\subsection{Shape based Methods}

To enhance the accuracy, shape- based segmentation techniques where the prior information about the lung shape is added to the image signal. [30] used nodule gradient to improve shape based segmentation. To segment ellipsoidal nodules, [31] recommended multi scale Gaussian intensity model. Active Shape model (ASM) was proposed by [32] to extract the lung fields. [33] and [34] suggested a way to use shape based model of a normal lung to extract a pathological lung. A graph based search method with the information about intensity, gradient and rib to automatically segment the lung was proposed by [35].

\subsection{Edge Based Approach}

The edge based segmentation algorithms extensively used edge detector filters and wavelet transforms. [36] used first derivative Gaussian filters to spot an outline of lung borders. Laplacian of Gaussian (LoG) operator was used to find a continuous lung contour which was combined together to extract the final lung region. Edge point detection method using spatial edge detection filters with closed contour for lung boundaries was proposed by [37].

\subsection{Atlas Based Approach}

Another novice approach to extract the lung fields is atlas based segmentation. An atlas is a composite image consists of segmented and co registered images of several objects. [33] used elastic registration toolbox to extract the lung with probabilistic atlas. Statistical atlas and graph cuts were used to enhance the segmentation precision by [38]. Table 2 presents the summary of various methods in Lung parenchyma segmentation and their performance. 
International Journal on Cybernetics \& Informatics (IJCI) Vol. 4, No. 1, February 2015

Table 2. Review of Lung Parenchyma Segmentation Methods on CT images

\begin{tabular}{|l|l|l|l|l|}
\hline Study & Method & 2D/3D & Dataset & Performance \\
\hline Hu et al.[25] & $\begin{array}{l}\text { Iterative threshold, } \\
\text { Morphological } \\
\text { Operations }\end{array}$ & 3D & $\begin{array}{l}\text { 24 datasets from } \\
8 \text { subjects }\end{array}$ & $\begin{array}{l}\text { Root mean square } \\
\text { Difference=0.54mm }\end{array}$ \\
\hline $\begin{array}{l}\text { Mendonca et } \\
\text { al.[37] }\end{array}$ & $\begin{array}{l}\text { Spatial edge } \\
\text { detector }\end{array}$ & 2D & $\begin{array}{l}47 \text { image } \\
\text { radiographs }\end{array}$ & $\begin{array}{l}\text { Sensitivity=92.25\% } \\
\text { Positive predictive } \\
\text { value=96.8\% }\end{array}$ \\
\hline Yim et al [26] & $\begin{array}{l}\text { Region growing, } \\
\text { Connected } \\
\text { component }\end{array}$ & 3D & 10 cases & $\begin{array}{l}\text { Root mean square } \\
\text { Difference=1.2 pixel }\end{array}$ \\
\hline $\begin{array}{l}\text { Sluimer et al. } \\
\text { [34] }\end{array}$ & Shape based & 3D & 26 cases & $\begin{array}{l}\text { Overlap Measure=81.65\% } \\
\text { Distance=1.48mm } \\
\text { Hausdorff } \\
\text { Distance=13.45mm }\end{array}$ \\
\hline $\begin{array}{l}\text { Campadelli et } \\
\text { al [36] }\end{array}$ & $\begin{array}{l}\text { Spatial Edge } \\
\text { Detector }\end{array}$ & 2D & $\begin{array}{l}487 \text { image } \\
\text { radiographs }\end{array}$ & $\begin{array}{l}\text { Sensitivity=91.74\% } \\
\text { Specificity=95.84\% } \\
\text { Positive Predictive } \\
\text { value=91.97\% } \\
\text { Accuracy=94.37\% }\end{array}$ \\
\hline Gao et al. [27] & Thresholding & 2D & 8 cases & $\begin{array}{l}\text { Dice Similarity } \\
\text { Coefficient=99.46\% }\end{array}$ \\
\hline Hua et al[35] & Graph-search & 3D & 15 cases & $\begin{array}{l}\text { Hausdorff } \\
\text { Distance=13.3pixel } \\
\text { Sensitivity=98.6\% } \\
\text { Specificity=99.5\% }\end{array}$ \\
\hline
\end{tabular}

\section{CANDidate Nodule Detection}

A pulmonary nodule is almost spherical shaped opacity measuring less than $3 \mathrm{~cm}$ in diameter surrounded by lung parenchyma. Its shape can be deformed by the neighbouring vessels or pleural surface. 4 types of nodules identified by [39] were,

(1) Well-circumscribed: The nodules is at the core of the lung tissue without being connected to vasculature.

(2) Vascularized: The nodule is at the centre of the lung filed but is significantly connected to the surrounding lung vessels.

(3) Pleural Tail: The nodule is located near the pleural surface, connected by a thin structure.

(4) Juxta pleural: A substantial portion of the nodule is connected by a thin structure.

Nodule detection is a process of identifying the nodules and their location in the lung field. The success of this process heavily depends on the accuracy of the lung parenchyma segmentation and false positive reduction method. Well-circumscribed nodules detection is relatively easy as they are isolated in nature. But the detecting the other three types is a challenging task as these types generate mostly false positive results.

Numerous methods have been proposed for detecting the candidate lung nodules. Thresholding, Morphological processing, template matching, adaptive thresholding, watershed algorithm, region 
growing approach, wave front algorithm, bounding box method, grey level thresholding, rolling ball algorithm, rule based method, clustering, connected component analysis and a combination of these methods have lead to other methods of segmentation.

\subsection{Thresholding}

A combination of thresholding and rule based method was proposed by [40] to identify the location of nodules. [41] used Bounding box method, gray level thresholding and rolling ball algorithm for segmenting lung nodules followed by a dot enhancement filter applied on three directions. Thresholding and multi scale morphological filtering were implemented by [42] to detect SPNs. [19] applied thresholding techniques followed by watershed algorithm and region growing technique to attain more accurate segmentation of nodules. Adaptive threshold technique was used on twice segmented lung filed to select candidate nodules by [43].

\subsection{Morphological Approach}

Morphological based segmentation was used by [44] to detect the pulmonary nodules. Non Juxta pleural nodules and Juxta pleural nodules were segmented from a huge set of CT scans by [45] using morphological operations which extracted both small and large nodules. [46] proposed the successive use of thresholding and morphological operations to extract SPNs.

\subsection{Template Matching}

Template matching methods to segment the SPNs were used by [47] [48] that could detect the circular /semicircular nodules. [49] developed both circular and semicircular templates to detect nodules residing inside and on the boundaries of lung region. This circular, spherical hypothesis is not enough to portray the actual geometry of nodules. Lesion's geometry can be irregular due to their attachment to the pleural surface or lung vessels. A variational level set segmentation was proposed by [50] [51] where a signed distance function was used to represent a $2 \mathrm{D}$ contour followed by template matching algorithm to extract juxta pleural nodules. [52] proposed an Active appearance Model (AAM) with template matching and registration to detect SPNs. Template based approach for the segmentation of nodules was implemented by [53].

\subsection{Other Methods}

Channeler ant model was used by [54] for segmenting candidate nodules. [55] adapted Kernel Rx Algorithm used in military imaging application to segment lung nodules. Median filters, morphological dilation and erosion filters were further used to improve the quality of segmentation. Summary of various approaches proposed by active researchers and their performances are presented in the Table 3 .

Table: 3 Literature survey of various Solitary Pulmonary Nodule detection approaches

\begin{tabular}{|l|l|l|l|l|l|l|}
\hline Study & Method & Dim & $\begin{array}{l}\text { No of } \\
\text { Cases }\end{array}$ & $\begin{array}{l}\text { Accuracy } \\
\text { (in \%) }\end{array}$ & $\begin{array}{l}\text { Sensitivity } \\
\text { (in \%) }\end{array}$ & $\begin{array}{l}\text { Specificity } \\
\text { (in \%) }\end{array}$ \\
\hline
\end{tabular}


International Journal on Cybernetics \& Informatics (IJCI) Vol. 4, No. 1, February 2015

\begin{tabular}{|c|c|c|c|c|c|c|}
\hline $\begin{array}{l}\text { Roozgard et } \\
\text { al.[55] }\end{array}$ & $\begin{array}{l}\text { Watershed Algorithm, } \\
\text { Median Filter, } \\
\text { Morphological Dilation } \\
\text { and Erosion Filter }\end{array}$ & $2 \mathrm{D}$ & 12 & -- & -- & -- \\
\hline $\begin{array}{l}\text { Si Guang-lei } \\
\text { et al.[45] }\end{array}$ & $\begin{array}{l}\text { Thresholding, } \\
\text { Morphological Processing }\end{array}$ & $2 \mathrm{D}$ & 85 & 90.5 & -- & -- \\
\hline $\begin{array}{l}\text { Takahiro et } \\
\text { al.[8] }\end{array}$ & $\begin{array}{l}\text { Thresholding, } \\
\text { Watershed Algorithm }\end{array}$ & $2 \mathrm{D}$ & 31 & 96.9 & -- & -- \\
\hline $\begin{array}{l}\text { Vijaya } \\
\text { Kishore et } \\
\text { al.[19] }\end{array}$ & $\begin{array}{l}\text { Thresholding, } \\
\text { Watershed Algorithm, } \\
\text { Region Growing } \\
\text { Segmentation }\end{array}$ & $2 \mathrm{D}$ & -- & -- & -- & -- \\
\hline $\begin{array}{l}\text { Maria } \\
\text { Evelina et } \\
\text { al.[54] }\end{array}$ & $\begin{array}{l}\text { 3D Region Growing } \\
\text { Method, } \\
\text { Wavefront Algorithm, } \\
\text { Morphological } \\
\text { Operations }\end{array}$ & $2 \mathrm{D}$ & 138 & -- & 80 & -- \\
\hline $\begin{array}{l}\text { Amal Farag } \\
\text { et al.[52] }\end{array}$ & Template Matching & $2 \mathrm{D}$ & 50 & -- & -- & -- \\
\hline $\begin{array}{l}\text { Mickias } \\
\text { Assefa et } \\
\text { al.[49] }\end{array}$ & Template Matching & $2 \mathrm{D}$ & 165 & 81.21 & -- & -- \\
\hline $\begin{array}{l}\text { Amal Farag } \\
\text { et al.[52] }\end{array}$ & $\begin{array}{l}\text { Template Matching, } \\
\text { Registration }\end{array}$ & $2 \mathrm{D}$ & 50 & -- & -- & -- \\
\hline $\begin{array}{l}\text { Hong Shao } \\
\text { et al. [43] }\end{array}$ & $\begin{array}{l}\text { Template Matching, } \\
\text { Adaptive Threshold } \\
\text { Technique }\end{array}$ & $2 \mathrm{D}$ & 130 & 90.35 & 89.47 & 90.52 \\
\hline $\begin{array}{l}\text { Hiram } \\
\text { Madero et } \\
\text { al.[66] }\end{array}$ & $\begin{array}{l}\text { Template Matching, } \\
\text { Watershed Algorithm }\end{array}$ & $2 \mathrm{D}$ & 61 & 82.66 & 96.15 & 52.17 \\
\hline $\begin{array}{l}\text { Amal A } \\
\text { Farag et } \\
\text { al.[51] }\end{array}$ & $\begin{array}{l}\text { Variational Level Set } \\
\text { Segmentation, } \\
\text { Template Matching }\end{array}$ & $2 \mathrm{D}$ & 50 & 70 & -- & -- \\
\hline $\begin{array}{l}\text { Wei Ying et } \\
\text { al.[42] }\end{array}$ & $\begin{array}{l}\text { Thresholding, } \\
\text { Morphological Filtering, } \\
\text { Boundary Extraction } \\
\text { Morphological Filtering }\end{array}$ & $2 \mathrm{D}$ & 20 & -- & 94.6 & -- \\
\hline $\begin{array}{l}\text { Yang Liu et } \\
\text { al.[41] }\end{array}$ & $\begin{array}{l}\text { Bounding Box Method, } \\
\text { Gray Level Thresholding, } \\
\text { Rolling Ball Algorithm, } \\
\text { Dot Enhancement Filter }\end{array}$ & $2 \mathrm{D}$ & 32 & -- & 94 & 92 \\
\hline $\begin{array}{l}\text { Wei Guo et } \\
\text { al.[42] }\end{array}$ & $\begin{array}{l}\text { Best Weight } \\
\text { Segmentation, } \\
\text { K-means Clustering and } \\
\text { Filtering }\end{array}$ & $2 \mathrm{D}$ & 29 & 90.73 & 94.77 & -- \\
\hline
\end{tabular}

\section{FAlSe Positive Reduction}


International Journal on Cybernetics \& Informatics (IJCI) Vol. 4, No. 1, February 2015

It is the process of eliminating false positive cases i.e. purge the nodule likes, incorrectly detected anatomical lung structure during candidate lung nodule detection process. Most of the CAD systems face problem because of relatively large number of false positives which affects the diagnosis, lowers the confidence of a radiologist using such a CAD scheme.

The methods mostly used for false positive reduction of nodules are rule based method, Neural Network classifiers, Selective enhancement filter, Support Vector Machines (SVM), Linear Discriminant Analysis (LDA) classifiers.

Rule based classifiers followed by artificial neural network classifiers were used to reduce FPs by [56]. [57] devised a highly nonlinear Massive Training Artificial Neural Network Classifier (MTANN) for false positive reduction of SPNs. Dot enhancement filters and neural network classifiers were used for FP reduction by [58]. Selective enhancement filters were used by [59] to reduce FPs by suppressing the vessel-like structure. SVM classifiers are used to by many researchers to enhance the true positives. [41] used 3 SVM classifiers to reduce FP's. A two stage method to address the false positive reduction was proposed by [60]. First, a rule based classifier was used for quick removal of FPs followed by Multiple Massive Training Support Vector Machine (MMTSVM) which clearly separates nodule and non-nodules.

A supervised classification method, LDA was used by [61]. Voxel (Volumetric pixel) based false positive reduction methods were used by [62] [63]. [49] extracted intensity based and multi resolution based features such as mean, energy, entropy etc. to reduce the false positive cases.

\section{Nodule Classification}

After the candidate nodules are detected and false positives are reduced, the resultant set of nodules must be classified to be benign or malignant ones. Most of the pulmonary nodules are benign but they may represent an early stage of lung cancer. Early detection of a malignant (cancerous) nodule increases the survival rate of the diseased. Many Computer Aided Diagnosis (CADx) systems have been developed that differentiate malignant lesions from benign ones, and also gives the insight into the proximity of detected nodule to be malignant. Receiver operating Curve (ROC) approach is used to evaluate the classifiers.

Despite the fact that the benign and malignant nodules have overlapping attributes, certain discriminating features such as morphological features, shape, size, appearance and growth rate of nodules makes the classification of nodule possible. Among these parameters growth rate of SPNs are regarded as an inventive clue for evaluating malignancy. [64] has listed various techniques are used for classification of SPNs such as Rule based classifiers, Markov Random Field, Neural Network, Bayesian classifier so on. The following sections reviews mostly used techniques for the categorization.

\subsection{Feature Based Classification:}

[65] concluded that a nodule having intense calcification, round shape regular boundary with lesser rate of growth has proximity to become benign. Higher growth rate, solid nodule with speculated outline classifies a nodule as malignant. Many statistical features, luminance features and Scale Invariant Feature Transform (SIFT) features were used by [8] for classification of nodules. [50] exercised nine similarity measures for categorization. [49] extracted intensity based and multi resolution based features to reduce the false positive cases. A mathematical model proposed by [39] which used the estimation of growth rate and volume doubling time to classify the nodules appropriately. 
International Journal on Cybernetics \& Informatics (IJCI) Vol. 4, No. 1, February 2015

\subsection{SVM based Classification:}

Classification in frequency domain using SVM classifier was performed by [43] [66]. [42] used SVM and weighted modifies Mahalanobis distance measure to nodule classification. Shape and histogram based analysis were used by [67]. A SVM with Gaussian kernel was used by [68] to classify the nodules. A mathematical model proposed by [39] which used the estimation of growth rate and volume doubling time to classify the nodules appropriately. Although several CAD schemes were able to deliver enhanced accuracy, detecting and characterizing Ground Glass Opacity (GGO) nodules would be the top priority of the researchers.

\section{CONCLuSion}

Designing an effective CAD systems for detection of lung nodules has been gaining its momentum as early detection increases the survival rate. In this paper a review of various approaches towards an automated detection of lung nodules, classifications are summarized. It is apparent from the review that the algorithms with multiple detection approaches provided the better results.

It is evident from the survey that the necessity to move from 2D to 3D CT image processing is more demanding. This inevitably brings more challenges in the processing steps such as segmentation. Designing algorithms to detect the nodules of different geometrical shapes and small ones needs further research. Nodules attached to the lung borders needs more sophisticated approach to be detected. Many of the techniques presented have reported more number of false positives. Reducing them further is one of the open challenges. Though most of the research works discussed so far emphasized more on final steps of the detection system, pre-processing steps deserve more attention towards optimization. Efficiency of the algorithms acceptable by the radiologists is another open issue to be addressed.

With the clinical importance of CT based detection and the challenges presented, it can be expected that $\mathrm{CAD} / \mathrm{x}$ of $\mathrm{CT}$ scans in the area of lung pathology will remain a challenging and an active research area in the years to come.

\section{REFERENCES}

[1] American cancer society, Cancer facts and figures, 2013.

[2] Heather R. Sanders, Maher Albitar, "Somatic mutations of signaling genes in non-small-cell lung cancer”, Cancer Genetics and Cytogenetics, 203, Elsevier, pp.7-15, 2010.

[3] M.V. Sprindzuk et al. "Lung cancer differential diagnosis based on the computer assisted radiology: The state of the art”, Polish journal of Radiology, Pol J Radiol, 75(1): 67-80, 2010.

[4] K.Devaki1 and V.MuraliBhaskaran, "Study of Computed Tomography Images of the Lungs: A Survey", IEEE-International Conference on Recent Trends in Information Technology, ICRTIT,pp.837842, 2011.

[5] Early Lung Cancer Action Program(ELCAP) : http://via.cornell.Edullungdb.html

[6] Lung Image Database Consortium(LIDC): https://imaging.nci.nih.gov/ncia/login.jsf

[7] Medical Image Database: MedPix : http://rad.usuhs.edu/medpix/index.html

[8] Takahiro Miyajima et al., "Classification of Lung Nodules Temporal Subtraction Image Based on Statistical Features and Improvement of Segmentation Accuracy”, proc. 12th IEEE International Conference on Control, Automation and Systems, Jeju Island, Korea, pp.1814-1817, 2012.

[9] H. Arimura, S. Katsuragawa, K. Suzuki, F. Li, F. Shiraishi, and S. Sone, et al., "Computerized scheme for automated detection of lung nodules in low-dose Computed Tomography images for lung cancer screening”, Acad. Radiology, vol. 11, pp. 617-29, 2004. 
International Journal on Cybernetics \& Informatics (IJCI) Vol. 4, No. 1, February 2015

[10] Farag, A.E. Baz, G. Gimelfarb, and R. Falk, "Automatic detection and recognition of lung abnormalities in helical CT images using deformable templates", Medical image Computing and Computer assisted Intervention-MICCAI, Springer, Berlin, vol. 3217, pp. 856-64, 2004.

[11] Sun, S.S., Li, H., Hou, X. R., et al., "Automatic segmentation of pulmonary nodules in CT images, IEEE”, 1st International Conference on Bioinformatics and Biomedical Engineering (ICBBE), pp. 790-793. 2007.

[12] Jia, T., Zhao, D.-Z., Yang, J.-Z., et al., "Automated detection of pulmonary nodules in HRCT images", IEEE, 1st International Conference on Bioinformatics and Biomedical Engineering (ICBBE), pp. 833-836. 2007.

[13] Chen et al., "Pulmonary micro nodule detection from 3D chest CT medical image", in MICCAI, vol. 3217, pp. 821-828. 2004.

[14] Sergei V. Fotin et al., "A multiscale Laplacian of Gaussian filtering approach to automated pulmonary nodule detection from whole-lung low-dose CT scans", Medical Imaging 2009, Proc. of SPIE Vol. 7260, pp. 72601Q-1-8,2009.

[15] Diciotti, S., Picozzi, G., Falchini, M., et al., "3D segmentation algorithm of small lung nodules in spiral CT images", IEEE Trans. Inf. Technol. Biomedical 12, pp, 7-19 2008.

[16] Sluimer, I.C., Van Waes, P.F., Vierever, M.A., et al., "Computer- aided diagnosis in high resolution CT of the lungs", Med. Phys. 30, pp, 3081-3090, 2003.

[17] S.Sivakumar et al., "Lung Nodule Detection Using Fuzzy Clustering and Support Vector Machines", International Journal of Engineering and Technology (IJET), vol. 5 No. 1, pp. 179-185, 2013.

[18] Jaesung et al., "Segmentation of Individual Ribs from Low-dose Chest CT", Proc. of SPIE Vol. 7624 , Medical Imaging 2010: Computer-Aided Diagnosis , pp. 76243J-1 -8, 2010

[19] V. Vijaya Kishore, R.V.S. Satyanarayana, "Performance Evaluation of Edge Detectors - Morphology Based ROI Segmentation and Nodule Detection from DICOM Lung Images in the Noisy Environment", proc. 3rd IEEE International Advanced Computing Conference, pp. 1131-1137, 2012.

[20] T. Oda, M. Kubo, Y. Kawata, N. Niki, K. Eguchi, and H. Ohmatsu, et al., "A detection algorithm of lung cancer candidate nodules on multi-slice CT images", in proc. of SPIE, vol. 4684, pp. 1354$61,2002$.

[21] Hong Shao et al., "A Detection Approach for Solitary Pulmonary Nodules Based on CT Images", proc. 2nd IEEE International Conference on Computer Science and Network Technology, pp. 12531257, 2012.

[22] Wook-Jin Choi et al., "Automated Pulmonary Nodule Detection System in Computed Tomography Images: A Hierarchical Block Classification Approach”, Entropy 2013, vol 5, pp. 508-523, 2013.

[23] William et al., "Automated volumetric segmentation method for growth consistency of nonsolid pulmonary nodules in high-resolution CT", proc. of SPIE, vol. 6514, Medical Imaging 2007: Computer-Aided Diagnosis, pp. 64150y0-10, 2007.

[24] Shingo et al., "Enhancement and detection of lung nodules with Multiscale filters in CT images", proc. International Conference on Intelligent Information Hiding and Multimedia Signal Processing, pp. 717-20, 2008.

[25] S. Hu, E. A. Hoffman, and J. M. Reinhardt, "Automatic lung segmentation for accurate quantitation of volumetric X-ray CT images,” IEEE Transactions on Medical Imaging, vol. 20, no. 6, pp. 490-498, 2001.

[26] Y. Yim, H. Hong, and Y. G. Shin," Hybrid lung segmentation in chest CT images for computer-aided diagnosis", in 7th International Workshop on Enterprise Networking and Computing in Healthcare Industry, HEALTHCOM2005, pp. 378-383, kor, June 2005.

[27] Q. Gao, S.Wang, D. Zhao, and J. Liu, "Accurate lung segmentation for X-ray CT images", in Proceedings of the 3rd International Conference on Natural Computation (ICNC '07), vol. 2, pp. 275279, 2007.

[28] Q.Wei, Y. Hu, G. Gelfand, and J. H.MacGregor, "Segmentation of lung lobes in high-resolution isotropic CT images", IEEE Transactions on Biomedical Engineering, vol. 56, no. 5, pp. 1383-1393, 2009.

[29] Y. Itai, H. Kim, S. Ishikawa et al., "Automatic segmentation of lung areas based on SNAKES and extraction of abnormal areas", in Proceedings of the 17th IEEE International Conference on Tools with Artificial Intelligence (ICTAI '05), pp. 377-381, November 2005.

[30] Zhao, B., Yankelevitz, D., Reeves, A., et al., "Two dimensional multi-criterion segmentation of pulmonary nodules on helical CT images", Med. Phys. 26, 889-895,1999 
International Journal on Cybernetics \& Informatics (IJCI) Vol. 4, No. 1, February 2015

[31] Okada, K., Comaniciu, D., Krishnan, A., "Robust anisotropic Gaussian fitting for columetric characterization of pulmonary nodules in multislice CT", IEEE Trans. Med. Imaging 24, 409- 423 2005.

[32] Tsai, A. Yezzi,W.Wells et al., "A shape-based approach to the segmentation of medical imagery using level sets”, IEEE Transactions on Medical Imaging, vol. 22, no. 2, pp. 137-154, 2003.

[33] C. Sluimer, M. Niemeijer, and B. Van Ginneken, "Lung field segmentation from thin-slice CT scans in presence of severe pathology", in Proceedings of the Progress in Biomedical Optics and ImagingMedical Imaging, pp. 1447-1455, February 2004.

[34] Sluimer, M. Prokop, and B.V. Ginneken, "Toward automated segmentation of the pathological lung in CT”, IEEE Trans. On Med. Imaging, vol. 24, no. 5, pp. 1025-38, Aug. 2005.

[35] P. Hua, Q. Song, M. Sonka, E. A. Hoffman, and J. M. Reinhardt, "Segmentation of pathological and diseased lung tissue in CT images using a graph-search algorithm", in Proceedings of the International Symposium on Biomedical Imaging (ISBI '11), pp. 2072-2075, 2011.

[36] P. Campadelli, E. Casiraghi, and D. Artioli, "A fully automated method for lung nodule detection from postero-anterior chest radiographs”, IEEE Transactions on Medical Imaging, vol. 25, no. 12, pp. 1588-1603, 2006.

[37] M. Mendonca, J. A. da Silva, and A. Campilho, "Automatic delimitation of lung fields on chest radiographs", in Proceedings of the International Symposium on Biomedical Imaging (ISBI '04), vol. 2, pp. 1287-1290, 2004.

[38] Shimizu , K. Nakagomi, T. Narihira, H. Kobatake, S. Nawano, and K. Shinozaki, et al., "Automated segmentation of 3D CT images based on statistical atlas and graph cuts", in proc. of MCV, pp. 12938. 2010.

[39] Kostis, W.J., Reeves, A.P., Yankelevitz, D.F., et al., "Three dimensional segmentation and growthrate estimation of small pulmonary nodules in helical CT images", IEEE Trans., Medical Imaging 22, pp.1259-1274,2003

[40] S.G. Armato, M.L. Giger, C.J. Moran, J.T. Blackburn, K. Doi, and H. MacMahon, “Computerized detection of pulmonary nodules on CT scans", Radio Graphics, vol. 19, pp. 1303-11, 1999.

[41] Yang Liu et al., "A Study of Pulmonary Nodule Detection in Three-Dimensional Thoracic CT Scans", proc. IEEE Second International Conference on Computer Modeling and Simulation, pp. 481-484, 2010.

[42] Wei Ying et al., "Autonomous Detection of Solitary Pulmonary Nodules on CT Images for ComputerAided Diagnosis", proc. IEEE Chinese Control and Decision Conference, pp. 4054-4059, 2011.

[43] Hong Shao et al., "A Detection Approach for Solitary Pulmonary Nodules Based on CT Images", proc. 2nd IEEE International Conference on Computer Science and Network Technology, pp. 12531257, 2012.

[44] J.M. Kuhnigk, V. Dicken, L. Bornemann, A. Bakai, D. Wormanns, and S. Krass, et al., "Morphological segmentation and partial volume analysis for volumetry of solid pulmonary lesions in thoracic CT scans”, IEEE Trans. On Medical Imaging, vol. 25, no.6, pp. 417-34, 2006.

[45] Si Guang-lei et al. , "A Novel Method for Lung Nodule Segmentation Based on CT Images", proc. 2nd IEEE International Conference on Applied Robotics for the Power Industry, pp. 826-830, 2012.

[46] T. Messay, R.C. Hardie, and S.K. Rogers, "A new computationally efficient CAD system for pulmonary nodule detection in CT imagery”, Medical Image Analysis, vol. 14, no. 3, pp. 390 - 406, 2010.

[47] Y. Lee, T. Hara, H. Fujita, S. Itoh, and T. Ishigaki, "Automated detection of pulmonary nodules in helical CT images based on an improved template-matching technique", IEEE Transactions on Medical Imaging, vol. 20, no. 7, pp. 595-604, 2001.

[48] R. Wiemker, P. Rogalla, A. Zwartkruis, and T. Blaffert, "Computer aided lung nodule detection on high resolution CT data in Medical Imaging”, Image Processing, vol. 4684 of Proc. SPIE, pp. 677688, 2002.

[49] Mickias Assefa et al., "Lung nodule Detection Using Multi Resolution Analysis", proc. IEEE, International Conference on Complex Medical Engineering, ICME, pp.457-461, 2013.

[50] Amal Farag et al., "Small - Size Lung Nodule Modeling and Detection with Clinical Evaluation", proc. IEEE Cairo International Biomedical Engineering Conference, Cairo, Egypt, pp. 44-47, 2012.

[51] Amal A. Farag et al., "Variational Approach for Segmentation of Lung Nodules", proc.18th IEEE International Conference on Image Processing, pp. 2157-2160, 2011.

[52] Amal Farag er.al. "An AAM Based Detection Approach of Lung Nodules from LDCT scans", IEEE, pp. 1040-1043, 2012. 
International Journal on Cybernetics \& Informatics (IJCI) Vol. 4, No. 1, February 2015

[53] A. Reeves, A. Chan, D. Yankelevitz, C. Henschke, B. Kressler, and W. Kostis, "On measuring the change in size of pulmonary nodules", IEEE Transactions on Medical Imaging, vol. 25, pp. 435-450, 2006.

[54] Maria Evelina et al., "Algorithms for automatic detection of lung nodules in CT scans", IEEE International Workshop on Medical Measurement and Applications, MEMEA,2011

[55] A Roozgard et al., "Malignant Nodule Detection on Lung CT Scan Images with Kernel RXalgorithm", proc. IEEE-EMBS International Conference on Biomedical and Health Informatics, pp. 499-502, 2012.

[56] Arimura, H., Katsuragawa, S., Suzuki, K., et al., "Computerized scheme for automated detection of lung nodules in low-dose computed tomography images for lung cancer screening”, Acad. Radiol. 11, pp.617-629, 2004

[57] Kenji Suzuki et al., "Computer-Aided Diagnostic Scheme for Distinction Between Benign and Malignant Nodules in Thoracic Low-Dose CT by Use of Massive Training Artificial Neural Network", IEEE Transactions on Medical Imaging, vol. 24, no. 9, pp.1138-1150,2005.

[58] S.L. Lee, A.Z. Kouzani, and E.J. Hu, "Random forest based lung nodule classification aided by clustering", Computerized Medical Imaging and Graphics, vol. 34, pp. 535-42, 2010.

[59] Q. Li, and K. Doi, "New selective enhancement tilter and its application for significant improvement of nodule detection on Computed Tomography", in proc. of SPIE, vol. 5370, pp. 1-9, 2004.

[60] Zhenghao Shi et al., "A Computer Aided Pulmonary Nodule Detection System Using Multiple Massive Training SVMs”, Appl. Math. Inf. Sci. 7, No. 3, pp-1165-1172, 2013.

[61] M.N. Gurcan, B. Sahiner and N. Petrick, "Lung nodule detection on thoracic Computed Tomography images: Preliminary evaluation of a computer-aided diagnosis system," Medical Physics, vol. 29, pp. 2552-8, 2002.

[62] Suzuki, K., Li, F., Li, Q., et al., "Comparison between 2D and 3D massive-training ANNs (MTANNs) in CAD for lung nodule detection on MDCT”, Int. J. Comput. Assist. Radiol. Surg. 1, pp.354- 355 ,2006

[63] Gori, I., Bellotti, R., Cerello, P, et al.: "Lung nodule detection in screening computed tomography", IEEE Nuclear Science Symposium Conference Record. IEEE (2006)

[64] I. Sluimer, A. Schilham, M. Prokop, and B. V. Ginneken, "Computer analysis of Computed Tomography scans of the lung: A Survey”, IEEE Trans. on Medical Imaging, vol. 25, pp 385-405, 2006.

[65] D. Wormanns, and S. Diederich, "Characterization of small pulmonary nodules by CT", Eur. Radiology, vol. 14, pp. 1380-91, 2004.

[66] Hiram Madero Orozco et al., "Lung Nodule Classification in Frequency Domain Using Support Vector Machines", proc. IEEE 11th International Conference on Information Sciences, Signal Processing and their Applications: Main Tracks, pp. 870-875, 2012.

[67] Y. Kawata, N. Niki, H. Ohmatsu, M. Kosumoto, R. Kakinuma, and K. Yamada, et al., "Pulmonary nodule classification based on nodule retrieval from 3D thoracic CT image database", SpringerVerlag; Berlin Heidelberg: pp. 838-46, 2004.

[68] Sheng Chen et al., "Computerized Detection of Lung Nodules by Means of Virtual Dual-Energy Radiography”, IEEE Transactions on Biomedical Engineering, vol. 60, no. 2, pp.369-378,2013. 
International Journal on Cybernetics \& Informatics (IJCI) Vol. 4, No. 1, February 2015

\section{AUTHORS}

Bhavanishankar K has received his M.Tech degree from NITK, Surathkal, India in 2007 and is pursuing his Ph.D from VTU, Belagavi, Karnataka, India. Currently he is working as Assistant Professor in Dept. of Computer Science and Enggat RNS Institute of Technology, Bengaluru, India. His research interest include Image processing and Pattern Classification.

Prof. M V Sudhamani has received M.Tech degree from KREC, Surathkal, India in 1996 and Ph.D from VTU, Belagavi, Karnataka, India in 2008. Currently she is working as Professor and Head in the Department of Information Science and Enggat RNS Institute of Technology, Bengaluru, India. She has 23 years of experience in teaching and research. Her research interests include Content Based Image Retrieval, Advanced Algorithms, and Image Processing. She has published more than 42 papers in International Conferences/Journals.
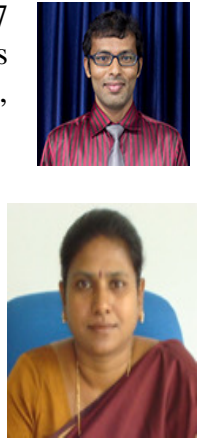